\title{
DIFFERENTIABLE SPHERE BUNDLES
}

\author{
S. P. NOVIKOV
}

The principal aim of this article is to prove that the connected components of the orthogonal group are not deformation retracts of the components of the group of diffeomorphisms of a sphere. The method employed also yields a number of other results.

\section{INTRODUCTION}

A fibration $p: V \rightarrow W$, where $V$ and $W$ are smooth manifolds and $p$ has maximal rank, equal to $\operatorname{dim} W$, at every point of $V$ will be called a twisted product (or bundle). The fiber $M=p^{-1}(x), x \in W$, is a smooth manifold and the structure group is in general the group $\operatorname{diff} M$ of smooth homeomorphisms of $M$. We shall study the special case when $M=S^{n-1}$ or $M=D^{n}$. It is known that when the fiber is $R^{n}$, the euclidean $n$-space, the bundle is equivalent to a linear bundle. This is so because diff $R^{n}$ has the same homotopy properties as the groups $S O_{n}$ and $G L(n)$. Until 1956 it seemed that this would also be the case for $S^{n-1}$. Then Milnor [5] constructed an example of a diffeomorphism $S^{6} \rightarrow S^{6}$ which was not smoothly isotopic to an orthogonal one. Thus the group diff $S^{n-1}$ generally has more connected components than the orthogonal group. Later Milnor, Kervaire and Smale (see [5, 8]) found quite a few components of diff $S^{n-1}$ which did not contain the components of $O_{n}$, but for $n \geq 4$ nothing was known about the relation between homotopy properties of $S O_{n}$ and the connected component $\operatorname{diff}^{0} S^{n-1}$ of the identity. The fact that $\operatorname{diff}^{0} S^{n-1}$ cannot be deformed onto $S O_{n}$ was first established by the author for $n=8$ in [6].

The present paper aims at a detailed exposition and further development of the previous work $[6]^{1}$ of this author. In $\S \S 4-5$ essential maps of spheres into $\operatorname{diff}^{0} S^{n-1}$ are constructed for some $n$; these maps cannot be deformed into the group $S O_{n}$. The existence of corresponding nonlinearizable sphere bundles over spheres of dimension $>1$ is then deduced (for the circle the existence of such bundles follows from Milnor's example of a nontrivial connected component of diff $S^{n-1}$ for $n=7)$. Results about homotopy properties of the spaces of smooth embeddings of a sphere in euclidian spaces are obtained as a byproduct (see $\S 5$ ). The results in $\S 1$ are not new, they are just an exposition of familiar and unfamiliar known facts in a form convenient for us. Among the results of $\S 2$ we note only the construction of

Translated by: S. Feder.

${ }^{1}$ The following corrections should be made to [6]:

1. In the table of homotopy groups of spheres replace $G_{14}=Z_{2}, \tilde{\theta}_{14}=0$ by $G_{14}=Z_{2}+Z_{2}$, $\tilde{\theta}_{14}=Z_{2}$.

2. In part 3) of Theorem 3 replace $A_{2 p-3,2 k p(p-1)-2 k} \otimes Z_{p} \supset Z_{p}+\cdots+Z_{p}(p-1$ terms $)$ by $A_{2 p-3,2 k p(p-1)-2 k} \otimes Z_{p} \supset Z_{p}$.

3. The author's footnote should be deleted. 
the homomorphisms $\lambda_{i}^{n}$ and $\mu_{i}^{n}$ and the relations between them. The main results are contained in $\S 4$ and also in $\S 5$ where consequences of $\S 4$ are deduced.

$\S 3$ contains, in a convenient form, well-known results of Milnor, Smale, Kervaire, Adams and others.

We note that the methods of this paper allow us to find nontrivial elements of $\pi_{i}\left(\operatorname{diff}^{0} S^{n-1}\right) / \pi_{i}\left(S O_{n}\right)$ only for $i<n / 2$. Presumably $\pi_{i}\left(\operatorname{diff}^{0} S^{n-1}\right) \approx \pi_{i}\left(S O_{n}\right)$ for large $i<n$. However, at this time the author has no way of proving it.

\section{$\S 1$. Differentiable Bundles. Classifying spaces}

Let $M$ be a $C^{\infty}$ smooth oriented compact manifold with or without boundary. We denote by $\operatorname{diff} M$ the group of all $C^{\infty}$ diffeomorphisms of $M$ with the topology which takes into account all derivatives (from zero to infinity). One can, as usual, consider bundles over finite complexes with fiber $M$ and structure group $\operatorname{diff} M$. This leads us to the usual concepts of associated, induced and universal bundles, which can be constructed for diff $M$ using the general theory. The base $B_{\operatorname{diff}} M$ of the universal bundle has the usual properties for bundles over finite complexes.

On the other hand, we may consider only those bundles for which the base and total space are smooth manifolds and the projection has maximal rank at each point (and is itself smooth of the same class as the space). We intend to give an interpretation of the universal bundle which will naturally distinguish the twisted products, just as in the case of a Lie group as the structure group.

Examples of bundles. 1 . $W \underset{p}{\stackrel{M}{\longrightarrow}} V$, where $W, V$ are smooth manifolds and $p$ has maximal rank (all of class $C^{\infty}$ ). If the manifolds $W, V, M$ are closed, no other conditions are necessary and the projection $p$ is a twisted product with structure group diff $M$. This is a typical example of a finite-dimensional differentiable bundle.

2. Let $\operatorname{diff}(M, W)$ denote the space of diffeomorphisms of $M$ into $W$. If $W$ has a boundary, we shall further assume that all diffeomorphisms have images strictly in the interior of $W$. On the space $\operatorname{diff}(M, W)$ we have actions without fixed points of $\operatorname{diff}(M)$ (on the left) and $\operatorname{diff}(W)$ (on the right). The factor space $\operatorname{diff}(M, W) / \operatorname{diff}(M)$ we shall denote by $P l(M, W)$, and call it the space of embeddings of $M$ into $W$ (in the naturally induced topology). We now establish some properties of the space $\operatorname{Pl}(M, W)$ and the projection $p: \operatorname{diff}(M, W) \rightarrow P l(M, W)$.

a. The space $\operatorname{Pl}(M, W)$ is locally contractible.

Proof. For simplicity assume that $M$ is closed and $f: M \subset W$ is some embedding, $f \in P l(M, W)$. For any sufficiently close embedding $g \in P l(M, W)$ we can find a perpendicular from a point $x \in g(M)$ to the embedded submanifold $f(M)$. This perpendicular is uniquely determined by $x$ and smoothly depends on $x$. This can be simultaneously done for all embeddings sufficiently close (in the $C^{2}$ sense) to the embedding $f$. A deformation of a small neighborhood $U(f) \subset P l(M, W)$ to the point $f$ can be given as follows: each point $x$ on the embedding $g(M)$ moves uniformly along its perpendicular with the speed equal to the length of the perpendicular from the point $x$ to the corresponding point on the embedding $f$. If $M$ is a manifold with boundary, then the proof easily generalizes, but first we must project not onto $f(M)$, but onto some smooth extension of it.

b. The projection $\operatorname{diff}(M, W) \rightarrow P l(M, W)$ has local sections. One considers the embedding $f \in P l(M, W)$, the diffeomorphism $\bar{f}: M \rightarrow W$ which gives rise 
to $f$ and extends it to the family of diffeomorphisms $U(f) \rightarrow \operatorname{diff}(M, W)$ using a deformation which is an inverse to the one described in a.

c. In the papers of Palais [7] and Cerf [2] a stronger fact was proven. Let diff $W$ act on the spaces $\operatorname{diff}(M, W)$ and $P l(M, W)$. Then for any point $\bar{f} \in \operatorname{diff}(M, W)$ there is a neighborhood $S(\bar{f}) \subset \operatorname{diff}(M, W)$ and a map $q: S(\bar{f}) \rightarrow \operatorname{diff}(W)$ such that $q(\bar{f})=e$ and $\bar{f} \circ q(\bar{g})=\bar{g}$. The group $\operatorname{diff}(W)$ acts on $\operatorname{diff}(M, W)$ on the right. Therefore the spaces $\operatorname{diff}(M, W)$ and $P l(M, W)$ are infinite-dimensional homogeneous spaces of diff $W$ with stationary group $D \subset \operatorname{diff} W$, consisting of maps leaving a given embedding $f: M \subset W$ pointwise fixed, and $D \times \operatorname{diff} M$ respectively.

Hence the projection $\operatorname{diff}(M, W) \rightarrow \operatorname{Pl}(M, W)$ is a fibration with structure group $\operatorname{diff} M$.

Consider the associated bundle $\nu_{W}$, with fiber $M$, base $P l(M, W)$ and projection $p$. The space of this bundle consists of pairs $(x, f)$ where $f \in P l(M, W)$ and $x \in W$ is a point on the embedding $f: M \subset W$.

Let $V$ be a smooth manifold and $f: V \rightarrow \operatorname{diff}(M, W)$ a continuous map. The map $f$ determines a map $f^{\prime}: V \times M \rightarrow V \times W$ by

$$
f^{\prime}(x, y)=(x, f(x) \circ y),
$$

where $f(x) \in \operatorname{diff}(M, W)$.

Definition 1. The map $f: V \rightarrow \operatorname{diff}(M, W)$ is called of class $C^{r}(r \geq 0)$ if the corresponding map $f^{\prime}: V \times M \rightarrow V \times W$ is of class $C^{r}$.

We have

Lemma 1. Every map $f: V \rightarrow \operatorname{diff}(M, W)$ of class $C^{r}(r \geq 0)$ can be approximated arbitrarily $C^{r}$-closely by a $C^{\infty}$ map $f_{1}: V \rightarrow \operatorname{diff}(M, W)$.

Proof. Consider the $C^{r}$ map $f^{\prime}: V \times M \rightarrow V \times W$. On each level $x \times M$ the map $f^{\prime}$ is of class $C^{\infty}$ and all differentials of order $<\infty$ continuously depend on the point $x \in V$. We approximate $f^{\prime}$ by a smooth map $f^{\prime \prime}: V \times M \rightarrow V \times W$ such that the restrictions $f^{\prime \prime} \mid x \times M$ and $f^{\prime} \mid x \times M$ are $C^{\infty}$-close diffeomorphisms $M \rightarrow V \times W$ for all $x$. The new map $f^{\prime \prime}$ does not preserve the cartesian product structure. Let us now project the image of $f^{\prime \prime} \mid x \times M$ onto the level $x \times W$ for all $x \in V$. This new map we denote by $f_{1}^{\prime}: V \times M \rightarrow V \times W$. This map is level preserving, is $C^{r}$-close to $f^{\prime \prime}$ and therefore to $f^{\prime}$, and is of class $C^{\infty}$. The map $f_{1}^{\prime}$ determines a smooth map $f_{1}: V \rightarrow \operatorname{diff}(M, W)$ which is $C^{\infty}$ by definition.

Let $q: \operatorname{diff}(M, W) \rightarrow P l(M, W)$ be the natural projection. Let $f: V \rightarrow \operatorname{diff}(M, W)$ and $q \circ f_{1}: V \rightarrow P l(M, W)$.

Definition 2. The map $q \circ f: V \rightarrow P l(M, W)$ is called smooth of class $C^{r}(r \geq 0)$ if there exists a lifting $f_{1}: V \rightarrow \operatorname{diff}(M, W)$ which is smooth of class $C^{r}$ and $q \circ f_{1}=$ $q \circ f$.

Lemma 1 trivially implies

Lemma 2. Every $C^{r}$ map $q \circ f: V \rightarrow P l(M, W)$ can be aproximated arbitrarily $C^{r}$-closely by a $C^{\infty}$ map $q \circ f_{1}$.

Thus we have a definition of smoothness for maps $g: V \rightarrow P l(M, W)$ which can be lifted. However, not all maps can be lifted. We shall use the fact that for the ball $D^{n}$, every map $g: D^{n} \rightarrow \operatorname{Pl}(M, W)$ can be lifted to a map $\bar{g}: D^{n} \rightarrow \operatorname{diff}(M, W)$. Thus for the ball $D^{n}$ we have the concept of a smooth map into $P l(M, W)$ and the approximation lemma. 
Definition 3. The map $g: V \rightarrow \operatorname{Pl}(M, W)$ is called smooth of class $C^{r}(r \geq 0)$ if for every point $x \in V$ there exists a ball-neighborhood $D_{x}^{n} \subset V$ containing the point $x$ in its interior, such that $g \mid D_{x}^{n}$ is smooth of class $C^{r}$.

Lemma 3. Every $C^{r}$-map $g: V \rightarrow P l(M, W)$ has an arbitrary close (in the $C^{r}$ sense) approximation $g_{1}: V \rightarrow P l(M, W)$ of class $C^{\infty}$.

Proof. For simplicity assume that $V$ is compact. Cover $V$ with a finite number of pairs of ball-neighborhoods $D_{i}^{n} \subset \tilde{D}_{i}^{n}$, so that every $x \in V$ lies in the interior of some $D_{i}^{n}$ and $D_{i}^{n}$ is concentrically included in $\tilde{D}_{i}^{n} \subset V, i=1, \ldots, m$. The map $g$ can be approximated so that it changes only in $\tilde{D}_{i}^{n}$ and becomes $C^{\infty}$ in a neighborhood of the ball $D_{i}^{n} \subset \tilde{D}_{i}^{n}$ for a given $i$. We can also assume that if $g$ was $C^{\infty}$ in some domain $U_{i} \subset \tilde{D}_{i}^{n}$, then in a smaller domain (which can be chosen to be as close to $U_{i}$ as we please) the approximation coincides with $g$. Putting these maps together we get, by induction, an approximation of $g: V \rightarrow P l(M, W)$ first in $\tilde{D}_{1}^{n}$, then in $\tilde{D}_{2}^{n}$, etc.

Remark. Any two close maps $g_{t}: V \rightarrow P l(M, W)$ are obviously homotopic; thus in Lemma 3 the maps $g$ and $g_{1}$ are homotopic.

From Lemma 3 we have

Lemma 4. 1) If $g: V \rightarrow P l(M, W)$ is a $C^{\infty}$ map then the induced bundle $g^{*} \nu_{W}$ with base $V$, total space $E_{g}$, fiber $M$ and projection $p_{g}$ has the following properties: a) $E_{g}$ is a smooth $C^{\infty}$ manifold; b) the projection $p_{g}$ has maximal rank at every point.

2) If $g_{t}: V \rightarrow P l(M, W), t=1,2$, are homotopic maps of class $C^{\infty}$, then there exists a $C^{\infty}$ diffeomorphism $f: E_{g_{1}} \rightarrow E_{g_{2}}$ commuting with the projections $p_{g_{1}}$ and $p_{g_{2}}$ (so $f$ leaves the base space pointwise fixed).

Proof. Let $g: V \rightarrow P l(M, W)$ be a $C^{\infty}$ map and $D_{i}^{n}$ be a closed (or open) ball. The bundle $g^{*} \nu_{W}$ is trivial over $D_{i}^{n}$ and there exists adiffeomorphism $f_{i}: D_{i}^{n} \times M \rightarrow$ $g^{*} \nu_{W}$, which is level preserving and identity on the base. This follows from the fact that the map on $D_{i}^{n}$ can be radially deformed into the constant map through $C^{\infty}$ maps. If the manifold $V$ is covered by balls $D_{i}^{n} \subset V$, then in the intersection $D_{i}^{n} \cap D_{j}^{n}$ we have a fiber map

$$
f_{i}^{-1} f_{j}:\left(D_{i}^{n} \cap D_{j}^{n}\right) \times M \rightarrow\left(D_{i}^{n} \cap D_{j}^{n}\right) \times M,
$$

which is a $C^{\infty}$ diffeomorphism. Therefore the total space $E_{g}$ of the bundle is covered by neighborhoods $D_{i}^{n} \times M$ with mappings $f_{i}^{-1} f_{j}$ which are diffeomorphisms. This proves 1 ). Note that the coordinate transformations induced by the maps $f_{i}^{-1} f_{j}$ are smooth maps $D_{i}^{n} \cap D_{j}^{n} \rightarrow \operatorname{diff} M$ in the sense of Definition 1 . To prove 2) we simply remark that two homotopic smooth maps are smoothly homotopic. Given this fact, the proof is just a repetition of the standard proof for $S O$-bundles.

We will next construct an $n$-universal bundle with diff $M$ as structure group. Let $m$ be the dimension of $M$. Let $W=R^{N}$ and consider the bundle

$$
\nu_{R^{N}}: T\left(M, R^{N}\right) \stackrel{M}{\longrightarrow} P l\left(M, R^{N}\right),
$$

where $N$ is a large integer.

Lemma 5. If $2(m+n)+3<N$, then $\pi_{i}\left(\operatorname{diff}\left(M, R^{N}\right)\right)=0$ for $i \leq n$. 
Proof. Consider an arbitrary map $g: S^{i} \rightarrow \operatorname{diff}\left(M, R^{N}\right)$ and extend it to a map of the ball $\tilde{g}: D^{i+1} \rightarrow \operatorname{diff}\left(M, R^{N}\right)$. First we approximate the map $g: S^{i} \rightarrow$ $\operatorname{diff}\left(M, R^{N}\right)$ by a $C^{\infty} \operatorname{map} g_{1}: S^{i} \rightarrow \operatorname{diff}\left(M, R^{N}\right)$ for which the map $\tilde{g}_{1}^{\prime}: S^{i} \times M \rightarrow$ $R^{N}$, where $\tilde{g}_{1}^{\prime}(x, y)=g_{1}(x) \cdot y$ and $g_{1}(x) \in \operatorname{diff}\left(M, R^{N}\right)$ is a $C^{\infty}$ embedding $S^{i} \times M \subset R^{N}$. Since $2(m+n)+3<N$ a theorem of Whitney allows us to do so. Further, we can extend the embedding $S^{i} \times M \subset R^{N}$ to an embedding $D^{i+1} \times M \subset R^{N}$ because of dimensional inequalities. Hence we extended the map $g_{1}$ to a smooth map of the ball $D^{i+1} \rightarrow \operatorname{diff}\left(M, R^{N}\right)$, but the maps $g$ and $g_{1}$ are homotopic.

Corollaries. 1. Since the bundle $\nu_{R^{N}}$ is associated with the principal bundle

$$
\operatorname{diff}\left(M, R^{N}\right) \stackrel{\operatorname{diff} M}{\longrightarrow} P l\left(M, R^{N}\right),
$$

it is n-universal for $N>2(m+n)+3$.

2. The bundle $\nu_{R^{N}}$ is also $n$-universal for differentiable bundles over smooth manifolds. Any differentiable bundle arises from a smooth homotopy class of maps $V \rightarrow \operatorname{Pl}\left(M, R^{N}\right)$ and vice versa.

3. Since continuous maps can be approximated by smooth ones, the smooth equivalence classes of smooth bundles coincide with usual equivalence classes of bundles.

4. If an embedding $f: W_{1} \rightarrow W_{2}$ is given, then we also have a bundle map $f_{*}: \nu_{W_{1}} \rightarrow \nu_{W_{2}}$. Letting $W_{2}=R^{N}$ we see that the bundle $\nu_{R^{N}}$ is also universal for "infinite-dimensional smooth bundles" $\nu_{W}$ constructed in the example 2 (see above), or at least if $N$ is sufficiently large, all these bundles embed in the universal one.

\section{$\S 2$. The main Structure groups and the Relations Between them}

We shall be interested mainly in the groups diff $M$ in the case when $M=S^{n-1}$, $D^{n}$ or $R^{n}$. Moreover, we will distinguish and study the following subgroups of those groups:

a) $S O_{n} \subset G L^{+}(n, R) \subset \operatorname{diff} S^{n-1}$.

b) $\Delta_{n}$, the group of those diffeomorphisms of the closed unit ball $D^{n}$, for each of which there exists $\epsilon>0$, depending on the diffeomorphism, such that in the $\epsilon$ neighborhood of the boundary the diffeomorphism takes a point with radius $1-\epsilon \leq$ $R \leq 1$ into a point with the same radius and a radial ray of length $\epsilon$ is mapped into a radial ray.

c) $K^{n}$, the group of diffeomorphisms of the closed ball $D^{n}$ leaving some neighborhood of its boundary pointwise fixed.

d) $K_{n}^{\prime}$, the group of diffeomorphisms of the closed ball $D^{n}$ leaving the boundary pointwise fixed.

As usual we denote by $G^{0}$ the connected component of the identity of a continuous group $G$.

Consider the following fibrations:

$$
\begin{gathered}
\operatorname{diff}^{0} D^{n} \frac{K_{n}^{\prime} \cap \operatorname{diff}{ }^{0} S^{n-1}}{p_{1}} \operatorname{diff}^{0} S^{n-1}, \\
\Delta_{n}^{0} \stackrel{K^{n} \cap \Delta_{n}^{0}}{p_{2}} \operatorname{diff}^{0} S^{n-1} .
\end{gathered}
$$


These give rise to the exact sequences

$$
\begin{gathered}
\rightarrow \pi_{i}\left(\operatorname{diff}^{0} D^{n}\right) \stackrel{p_{1 *}}{\longrightarrow} \pi_{i}\left(\operatorname{diff}^{0} S^{n-1}\right) \stackrel{\partial}{\rightarrow} \pi_{i-1}\left(K_{n}^{\prime} \cap \operatorname{diff}^{0} D^{n}\right), \\
\rightarrow \pi_{i}\left(\Delta_{n}^{0}\right) \stackrel{p_{2 *}}{\longrightarrow} \pi_{i}\left(\operatorname{diff}^{0} S^{n-1}\right) \stackrel{\partial}{\rightarrow} \pi_{i-1}\left(K^{n} \cap \Delta_{n}^{0}\right) .
\end{gathered}
$$

For the inclusions $\Delta \subset \operatorname{diff} D^{n}$ and $K^{n} \subset K_{N}^{\prime}$ we have

Lemma 6. The inclusions $\Delta_{n} \subset \operatorname{diff} D^{n}$ and $K^{n} \subset K_{n}^{\prime}$ induce isomorphisms on homotopy and homology groups of all dimensions.

Proof. We see from the fibrations (I) and (II) that it suffices to prove the lemma for the inclusion $K^{n} \subset K_{n}^{\prime}$. Let $f: S^{l} \rightarrow K_{n}^{\prime}$ be a map. We shall show that $f$ can be deformed into $K^{n}$. The map $f$ induces a map

$$
f: S^{l} \times D^{n} \rightarrow S^{l} \times D^{n},
$$

where $f^{\prime}(x, y)=(x, f(x) \circ y)$ as in $\S 1$. If $f$ is smooth (which we can assume without loss of generality) then $f^{\prime}$ is a diffeomorphism. The diffeomorphism $f^{\prime}$ (of class $C^{r}$ for sufficiently large $r$ ) leaves the boundary $S^{l} \times S^{n-1}$ fixed and the vector field on $S^{l} \times D^{n}$ which is normal to the boundary gets "bent" in some way, but the resulting field will be a field of smooth curves in $S^{l} \times D^{n}$, transversal to $S^{l} \times S^{n-1}$. Since this field is transversal and smooth, one can find a smooth fiber map which is an isotopy of this field with the field of normals; this isotopy will give a smooth homotopy of the map $f: S^{l} \rightarrow K_{n}^{\prime}$. The resulting map $\tilde{f}_{1}: S^{l} \rightarrow K_{n}^{\prime}$ determines a diffeomorphism

$$
\tilde{f}_{1}^{\prime}: S^{l} \times D^{n} \rightarrow S^{l} \times D^{n}
$$

which maps, in a sufficiently small neighborhood of the boundary, a radius normal to the boundary into itself. By a fiber-preserving isotopy we change this diffeomorphism into one that does not change the distance from the boundary if it is sufficiently small. This last diffeomorphism determines a map $f_{1}: S^{l} \rightarrow K_{n}^{\prime}$ which is actually a map into $K^{n}$. Analogously one proves that if a map $f: S^{l} \rightarrow K^{n}$ is null-homotopic in $K_{n}^{\prime}$ then that homotopy can be deformed into $K^{n}$ (the homotopy is a map $F: D^{l+1} \rightarrow K_{n}^{\prime}$ such that $\left.F \mid \partial D^{l+1}=f\right)$.

We proved thus that the inclusion $K^{n} \subset K_{n}^{\prime}$ induces isomorphisms on the homotopy groups. Since both spaces are of simple homotopy type, the lemma follows.

Lemma 6 implies that the exact sequences $\left(\mathrm{I}^{\prime}\right)$ and $\left(\mathrm{II}^{\prime}\right)$ actually coincide. Therefore from a homotopy standpoint the groups $\Delta_{n}$ and diff $D^{n}$ as well as $K^{n}$ and $K_{n}^{\prime}$ coincide. Moreover, it was shown in [10] that the homotopy and homology groups of diff $R^{n}$ are isomorphic to those of the subgroup $S O_{n}$ and the isomorphisms are given by the induced inclusion homomorphisms. ${ }^{2}$ It is well known that the group $G L^{+}(n, R)$ can be deformed onto $S O_{n}$.

It was also proven in [10] that the inclusion $S O_{n} \subset \operatorname{diff}^{0} S^{n-1}$ induces monomorphisms (this fact has an elementary proof).

Milnor has shown that the group $\pi_{0}\left(\operatorname{diff} S^{n-1}\right)$ is commutative. He has studied the group

$$
\pi_{0}\left(\operatorname{diff} S^{n-1}\right) / p_{1 *} \pi_{0}\left(\operatorname{diff} D^{n}\right)=\Gamma^{n}
$$

and has shown that $\Gamma^{7}$ is nontrivial (see [5]). Milnor has also shown that the elements of $\Gamma^{n}$ are in one to one correspondence with smooth structures on $S^{n}$ which have a smooth triangulation combinatorialy equivalent to the usual one. At

\footnotetext{
${ }^{2}$ This is proven for the $C^{1}$-topology.
} 
the present time it is known (see $[5,8,9]$ ) that the groups $\Gamma^{n}$ are finite for $n \neq 4$ and $\Gamma^{i}=0$ for $i \leq 3$.

It is also known that the groups diff $S^{1}$ and diff $S^{2}$ have $\mathrm{SO}_{2}$ and $\mathrm{SO}_{3}$ correspondingly as deformation retracts. There is no similar result for the group diff $S^{3}$. Smale has conjectured that $\mathrm{SO}_{4}$, is a deformation retract of diff $S^{3} .^{3}$

We shall study the groups diff $S^{n-1}$ for large $n$. So far, nothing has been known about the groups $\pi_{i}$ (diff $S^{n-1}$ ) for $i>0$ except for the facts mentioned above. We aim to prove that the groups $\pi_{i}\left(\operatorname{diff} S^{n-1}\right) / p_{1 *} \pi_{i}\left(\operatorname{diff} D^{n}\right)$ are nontrivial for $i>0$ and some values of $n$.

To study the groups $\pi_{i}\left(\operatorname{diff}^{0} S^{n-1}\right)$ we shall construct a homomorphism

$$
\lambda_{i}^{n}: \pi_{i}\left(\operatorname{diff}^{0} S^{n-1}\right) \rightarrow \pi_{0}\left(\operatorname{diff} S^{n+i-1}\right) .
$$

Let an element $\alpha \in \pi_{i}\left(\operatorname{diff}^{0} S^{n-1}\right)$ be represented by a smooth $C^{\infty}$ map

$$
f: D^{i} \rightarrow \operatorname{diff}^{0} S^{n-1},
$$

which takes a neighborhood of the boundary $\partial D^{i}=S^{i-1}$ into the identity of the group. The map $f$ in the usual way determines the diffeomorphism

$$
f^{\prime}: D^{i} \times S^{n-1} \rightarrow D^{i} \times S^{n-1},
$$

which leaves a neighborhood of the boundary $S^{i-1} \times S^{n-1}$ pointwise fixed. We can represent the sphere $S^{i+n-1}$ in the following way:

$$
S^{i+n-1}=S^{i-1} \times D^{n} \cup D^{i} \times S^{n-1} .
$$

On the manifold $D^{i} \times S^{n-1}$ we have the diffeomorphism $f^{\prime}$ which leaves a neighborhood of the boundary pointwise fixed. We extend this diffeomorphism over the sphere $S^{n+i-1}$ by letting it be the identity on $S^{i-1} \times D^{n}$. Let $\lambda_{i}^{n}(\alpha)$ be the smooth isotopy class of the constructed diffeomorphism. This way we get the map

$$
\lambda_{i}^{n}: \pi\left(\operatorname{diff} S^{n-1}\right) \rightarrow \pi_{0}\left(\operatorname{diff} S^{i+n-1}\right) .
$$

We shall prove that $\lambda_{i}^{n}$ is a well-defined homomorphism.

1. $\lambda_{i}^{n}$ is well-defined. Let $f$ and $g: D^{i} \rightarrow \operatorname{diff}^{0} S^{n-1}$ be $C^{\infty}$ maps representing the same element $\alpha \in \pi_{i}\left(\operatorname{diff}^{0} S^{n-1}\right)$ and taking a neighborhood of the boundary of $D^{1}$ into the identity of the group. We have then a smooth mapping of the cylinder

$$
F: D^{i} \times I \rightarrow \operatorname{diff}^{0} S^{n-1}
$$

such that $F\left|D^{i} \times 0=f, F\right| D^{i} \times 1=g$ and $F$ takes a neighborhood of $S^{i-1} \times I \subset$ $D^{i} \times I$ into the identity of the group. The mapping $F$ determines a diffeomorphism

$$
F^{\prime}: D^{i} \times I \times S^{n-1} \rightarrow D^{i} \times I \times S^{n-1}
$$

The diffeomorphism $F^{\prime}$ just as in the construction of $\lambda_{i}^{n}$ determines a diffeomorphism

$$
\bar{F}^{\prime}: S^{i+n-1} \times I \rightarrow S^{i+n-1} \times I
$$

for which $\left.\bar{F}^{\prime}\right|_{t=0}=\lambda_{i}^{n}(f),\left.\bar{F}^{\prime}\right|_{t=1}=\lambda_{i}^{n}(g)$ and the diffeomorphism $\bar{F}^{\prime}$ takes the point $(x, t)$ into the point $(y, t)$ where $t \in I$. Thus the diffeomorphisms $\lambda_{i}^{n}(f)$ and $\lambda_{i}^{n}(g)$ are isotopic, which shows that $\lambda_{i}^{n}$ is well-defined.

2. $\lambda_{i}^{n}$ is a homomorphism. Let $\alpha, \beta \in \pi_{i}\left(\operatorname{diff}^{0} S^{n-1}\right)$ and let $f, g: D^{i} \rightarrow$ $\operatorname{diff}^{0} S^{n-1}$ be maps representing $\alpha, \beta$ which take a neighborhood of the boundary of $D^{i}$ into the identity of the group. Assume that for each $x \in D^{i}$ either

\footnotetext{
${ }^{3}$ Very recently J. Cerf announced that $\pi_{0}\left(\operatorname{diff} S^{3}\right)=0$.
} 
$f(x)=e$ or $g(x)=e$ (i.e., the carriers of $f$ and $g$ do not intersect). It follows that the diffeomorphisms $\lambda_{i}^{n}(f)$ and $\lambda_{i}^{n}(g)$ commute and

$$
\lambda_{i}^{n}(f g)=\lambda_{i}^{n}(f) \lambda_{i}^{n}(g)
$$

which in turn implies that $\lambda_{i}^{n}$ is a homomorphism.

Consider the group $K^{n}$, consisting of diffeomorphisms of the ball $D^{n}$ leaving a neighborhood of the boundary fixed. We can define an inclusion $\varkappa: K^{n} \subset \operatorname{diff} S^{n}$ as follows: any diffeomorphism of the ball $D^{n}$ determines a diffeomorphism of $S^{n}$ if we consider $D^{n}$ to be the lower hemisphere of $S^{n}$. In the exact sequences $\left(\mathrm{I}^{\prime}\right)$ and $\left(\mathrm{II}^{\prime \prime}\right)$ the boundary homomorphism

$$
\partial: \pi_{i}\left(\operatorname{diff}^{0} S^{n-1}\right) \rightarrow \pi_{i-1}\left(K^{n} \cap \Delta_{n}^{0}\right)
$$

is determined.

We have the following diagram (for $i=1$ )

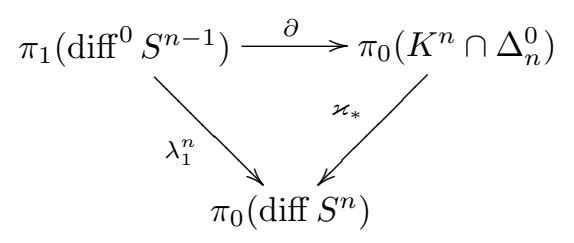

Lemma 7. The diagram (III) is commutative.

Proof. Consider the mapping $f: I \rightarrow \operatorname{diff}^{0} S^{n-1}$, taking a neighborhood of the boundary $\partial I=S^{0}$ into the identity of the group. This mapping determines a diffeomorphism

$$
f^{\prime}: S^{n-1} \times I \rightarrow S^{n-1} \times I
$$

for which $f^{\prime}(x, t)=\left(f_{t} \circ x, t\right)$. Extending this map to a diffeomorphism $S^{n} \rightarrow S^{n}$ we obtain the homomorphism $\lambda_{1}^{n}$. We now lift the map $f: I \rightarrow \operatorname{diff}^{0} S^{n-1}$ to a map $g: I \rightarrow \Delta_{n}^{\circ}$ such that $p_{2} \circ g=f$ and $g(0)=e$. We obtain a diffeomorphism

$$
g^{\prime}: D^{n} \times I \rightarrow D^{n} \times I,
$$

for which $g^{\prime} \mid D^{n} \times 0=1$. On the boundary

$$
g^{\prime} \mid S^{n-1} \times I=f^{\prime} .
$$

We attach a ball $D_{0}^{n}$ to $D^{n} \times I$ by identifying $\partial D_{0}^{n}$ with $D^{n} \times 1$ in the natural way. This determines diffeomorphisms representing $\varkappa_{*} \partial$ on the sphere

$$
S_{0}^{n}=D_{0}^{n} \cup D^{n} \times 1
$$

and $\lambda_{1}^{n}$ on the sphere

$$
S_{1}^{n}=D_{0}^{n} \cup \partial D^{n} \times I \cup D^{n} \times 0
$$

Let

$$
S_{t}^{n}=D_{0}^{n} \cup \partial D^{n} \times I(1-t, 1) \cup D^{n} \times(1-t),
$$

where $0 \leq t \leq 1$. It is easily seen that for $t=0,1$ we get the spheres $S_{0}^{n}, S_{1}^{n}$ as determined above. On the sphere $S_{t}^{n}$ we have the mapping $f_{t}: S_{t}^{n} \rightarrow S_{t}^{n}$ which leaves $D_{0}^{n} \subset S_{t}^{n}$ pointwise fixed and equals $g^{\prime}=f^{\prime} \mid \partial D^{n} \times I(1-t, 1)$ and equals $g^{\prime} \mid D^{n} \times(1-t)$. The only place where $f_{t}$ is not smooth is $\partial D^{n} \times(1-t)-$ we have a corner which can be easily smoothed. The maps $f_{t}$ give an isotopy between $f_{0}=\kappa_{*} \partial(f)$ and $f_{1}=\lambda_{1}^{n}(f)$. By smoothing the angles this isotopy is made to go 
through diffeomorphisms of $S^{n}$. Thus on homotopy classes $\kappa_{*} \partial=\lambda_{1}^{n}$. This proves the lemma.

Analogously with $\lambda_{i}^{n}$ we define homomorphisms

$$
\mu_{i}^{n}: \pi_{i}\left(\operatorname{diff}^{0} S^{n-1}\right) \rightarrow \pi_{i-1}\left(\operatorname{diff} S^{n}\right) .
$$

Let $\alpha \in \pi_{i}\left(\operatorname{diff}^{0} S^{n-1}\right)$ and consider the map $f: I^{i} \rightarrow \operatorname{diff}^{0} S^{n-1}$ of the cube, which takes a neighborhood of its boundary $\partial I^{i}$ into the identity of the group and which represents the class $\alpha$. The map

$$
f: I^{i} \rightarrow \operatorname{diff}^{0} S^{n-1}
$$

can be viewed as a family of mappings

$$
g_{t}: I^{i-1} \rightarrow \operatorname{diff}^{0} S^{n-1} .
$$

The smooth family $g_{t}$ defines a mapping

$$
\bar{g}: I^{i-1} \rightarrow \operatorname{diff}^{0}\left(S^{n-1} \times I\right),
$$

where $\bar{g}(x)(y, t)=\left(g_{t}(x), t\right)$. Diffeomorphisms in $\operatorname{Im} \bar{g} \subset \operatorname{diff}^{0}\left(S^{n-1} \times I\right)$ have natural extensions to diffeomorphisms of the sphere

$$
S^{n}=D_{0}^{n} \cup S^{n-1} \times I \cup D_{1}^{n} .
$$

This results in a mapping

$$
\mu_{i}^{n}: \pi_{i}\left(\operatorname{diff}^{0} S^{n-1}\right) \rightarrow \pi_{i-1}\left(\operatorname{diff} S^{n}\right),
$$

which generalizes the mapping

$$
\lambda_{1}^{n}: \pi_{1}\left(\operatorname{diff}^{0} S^{n-1}\right) \rightarrow \pi_{0}\left(\operatorname{diff} S^{n}\right) .
$$

We have indeed $\lambda_{1}^{n}=\mu_{1}^{n}$. Analogously with $\lambda_{i}^{n}$ we get the following properties of $\mu_{i}^{n}$ :

1. The mappings $\mu_{i}^{n}$ are well-defined homomorphisms.

2. The diagram

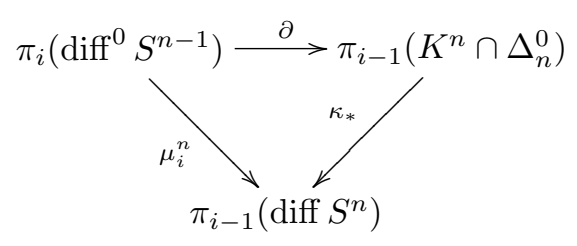

is commutative (the proof is as in Lemma 7).

3. The composition $\mu_{1}^{n+i-1} \circ \cdots \circ \mu_{i}^{n}=\lambda_{i}^{n}$.

The statements 1 and 2 have been proven in special cases, and the general proof is identical to the ones given previously. The proof of 3 follows easily from the construction of the homomorphisms $\lambda_{i}^{n}$ and $\mu_{i}^{n}$.

Let us draw some conclusions from the results of this section. We have established that from a homotopy point of view the groups $\Delta_{n}$ and diff $D^{n}$, as well as $K^{n}$ and $K_{n}^{\prime}$ coincide (the author does not know whether the proof of this obvious 
fact is contained in the literature). Moreover, we defined the homomorphisms

$$
\begin{aligned}
\lambda_{i}^{n}: \pi_{i}\left(\operatorname{diff}^{0} S^{n-1}\right) & \rightarrow \pi_{0}\left(\operatorname{diff} S^{n+i-l}\right), \\
\mu_{i}^{n}: \pi_{i}\left(\operatorname{diff}^{0} S^{n-1}\right) & \rightarrow \pi_{i-1}\left(\operatorname{diff} S^{n}\right), \\
\kappa_{*}: \quad \pi_{i-1}\left(K^{n}\right) & \rightarrow \pi_{i-1}\left(\operatorname{diff} S^{n}\right), \\
\partial: \pi_{i}\left(\operatorname{diff}^{0} S^{n-1}\right) & \rightarrow \pi_{i-1}\left(K^{n} \cap \Delta_{n}^{0}\right),
\end{aligned}
$$

and proved that

$$
\mu_{i}^{n}=\kappa_{*} \partial, \quad \mu_{1}^{n}=\lambda_{1}^{n}, \quad \lambda_{i}^{n}=\mu_{1}^{n+i-1} \circ \cdots \circ \mu_{i}^{n} .
$$

The properties of the homomorphisms (IV) imply that

$$
p_{1 *} \pi_{i}\left(\operatorname{diff}^{0} D^{n}\right) \subset \operatorname{Ker} \mu_{i}^{n} \subset \operatorname{Ker} \lambda_{i}^{n} .
$$

Hence the homomorphisms $\lambda_{i}^{n}$ and $\mu_{i}^{n}$ are well-defined on the quotient groups

$$
\pi_{i, n}=\pi_{i}\left(\operatorname{diff}^{0} S^{n-1}\right) / p_{1 *} \pi_{i}\left(\operatorname{diff} D^{n}\right), \quad i>0 .
$$

The groups $\pi_{i, n}$ will be the ones we will be interested in.

\section{$\S 3$. Homotopy groups of spheres and the groups of Milnor}

In this section, for convenience of further exposition, we shall give the known definitions and interrelations for the groups $\Gamma^{n}, \theta^{n}$ of Milnor and the homotopy groups of spheres $\pi_{N+n}\left(S^{N}\right)=G_{n}$.

The group $\Gamma^{n}$ is by definition the quotient

$$
\Gamma^{n}=\pi_{0}\left(\operatorname{diff} S^{n-1}\right) / \operatorname{Im} p_{1 *} .
$$

The group $\theta^{n}$ is constructed as follows: the elements are the $h$-cobordism classes of smooth oriented manifolds which are homotopy $n$-spheres, the composition is given by the connected sum of oriented manifolds and the identity is provided by the class of the ordinary $n$-sphere $S^{n}$. In the group $\theta^{n}$ there is a subgroup $\theta^{n}(\partial \pi)$, elements of which can be represented by homotopy spheres which bound $\pi$-manifolds. We have

Theorem (Adams). Every homotopy sphere is a $\pi$-manifold.

There is a homomorphism defined by Milnor on

$$
\delta: \theta^{n} \rightarrow G_{n} / \operatorname{Im} J, \text { where } I: \pi_{n}\left(S O_{N}\right) \rightarrow \pi_{n+N}\left(S^{N}\right), \quad \operatorname{Im} \delta=\tilde{\theta}^{n},
$$

which is easily defined through framed manifolds of L. S. Pontrjagin.

Theorem (Milnor). The kernel of the homomorphism $\delta$ is given by $\theta^{n}(\partial \pi)$.

This gives us an inclusion $\delta: \theta^{n} / \theta^{n}(\partial \pi) \subset G_{n} / \operatorname{Im} J$.

Let us list the results about $\theta^{n}$.

Theorem (Milnor, Kervaire). The groups $\theta^{2 k}(\partial \pi)$ are trivial. The groups $\theta^{4 k+1}(\partial \pi)$ contain not more than two elements, while

$$
\begin{gathered}
\theta^{1}(\partial \pi)=\theta^{5}(\partial \pi)=\theta^{13}(\partial \pi)=0, \\
\theta^{9}(\partial \pi)=\theta^{17}(\partial \pi)=Z_{2} .
\end{gathered}
$$

The groups $\theta^{4 k-1}(\partial \pi)$ are nontrivial cyclic of finite order for $k \geq 2$, while $\theta^{7}(\partial \pi)=$ $Z_{28}$ and $\theta^{11}(\partial \pi)=Z_{992}$. For $n \neq 4 k+2$,

$$
\delta\left(\theta^{n} / \theta^{n}(\partial \pi)\right)=G_{n} / \operatorname{Im} J .
$$


For $n=4 k+2$ the index of $\operatorname{Im} \delta$ in $G_{n} / \operatorname{Im} J$ does not exceed two; it is equal to two for $k=0,1,3$ and is equal to one for $k=2,4$.

Corollary (Milnor, Kervaire). The groups $\theta^{n}(n \neq 3)$ are finite. Moreover, there is a homomorphism $S: \Gamma^{n} \rightarrow \theta^{n}$, which is constructed as follows: let $h: S^{n-1} \rightarrow S^{n-1}$ be a diffeomorphism which represents an element $\alpha \in \Gamma^{n}$. Let $S(\alpha)=\left\{D^{n} \cup_{h} D^{n}\right\}$, where the curly brackets denote the h-cobordism class of the manifold $D^{n} \cup_{h} D^{n}$.

Theorem (Smale). ${ }^{4}$ The homomorphism $S: \Gamma^{n} \rightarrow \theta^{n}$ is a canonical isomorphism for $n \neq 3,4$. The elements of $\theta^{n}$ are smooth manifolds which are combinatorialy equivalent to the standard sphere $S^{n}$ and can be obtained by gluing together two balls $D^{n} \cup_{h} D^{n}$, where $h: S^{n-1} \rightarrow S^{n-1}$ determines uniquely an element of $\Gamma^{n}$.

Thus for $n \neq 4 k+2$ any element of $G_{n}$ can be represented by a framed manifold, combinatorially equivalent with $S^{n}$, and for $n=4 k+2$ at least half the elements of $G_{n}$ (those lying in $\operatorname{Im} \delta$ ) admit such a representation. Moreover, this smooth sphere can be obtained by identifying the boundaries of two balls using the diffeomorphism $h: S^{n-1} \rightarrow S^{n-1}$, where $h$ determines the unique element of the group $\Gamma^{n}$, which depends for $n \geq 5$, modulo $\theta^{n}(\partial \pi)$, only on the element of the group $G_{n} / \operatorname{Im} J$.

\section{$\S 4$. The STABLE HOMOtopy RING AND the GROUP OF DIFFEOMORPHISMS OF THE SPHERE}

It is well known that the direct $\operatorname{sum} G=\sum_{i \geq 0} G_{i}$, of the stable homotopy groups of spheres is an anticommutative graded ring. The multiplication is given by the cartesian product of framed manifolds. Let $J \subset G$ be the subgroup $J=\sum_{i \geq 0} J_{i}$, where $J_{i}=J \pi_{i}(S O)$. For our purposes, as we shall see later, we must know the multiplication $J \circ G$. Let us first establish some properties of the subgroup $J \subset G$. Let $M^{n}$ be a $\pi$-manifold.

Denote by $B\left(M^{n}\right) \subset G_{n}$ the set of elements of $G_{n}$ which can be represented by a framing of $M^{n}$ in the sense of Pontrjagin.

Lemma 8. $B\left(S^{i} \times S^{j}\right)=J_{i+j}$ except for $i=j=1,3,7$.

Proof. Let $\alpha \in B\left(S^{i} \times S^{j}\right)$ and let $i \leq j$. Consider the cycle $S^{i} \times x_{0} \subset S^{i} \times S^{j}$, where $x_{0} \in S^{j}$. Let $h: S^{i} \rightarrow S O_{j}$ be a smooth map and $h^{\prime}: S^{i} \times D^{j} \rightarrow S^{i} \times D^{j}$ a diffeomorphism for which

$$
h^{\prime}(x, y)=(x, h(x) \circ y) .
$$

We can prove by standard methods that the map $h: S^{i} \rightarrow S O_{j}$ for $i \leq j$ can be chosen so that the framing of $S^{i} \times S^{j}$ can be extended over the manifold

$$
B^{n+1}(h)=\left(S^{i} \times S^{j}\right) \times I(0,1 / 2) \cup_{h^{\prime}} D^{i+1} \times D^{j},
$$

where $h^{\prime}: \partial D^{i+1} \times D^{j} \rightarrow S^{i} \times S^{j} \times 1 / 2$ (for proof see $[5,6]$ ).

Let

$$
M^{n}(h)=\left(S^{i} \times S^{j} \backslash S^{i} \times D^{j}\right) \cup_{h^{\prime}} D^{i+1} \times \partial D^{j} .
$$

We easily see that $\partial B^{n+1}(h)=S^{i} \times S^{j} \cup M^{n}(h)$ and the manifold $M^{n}(h)$ is a homotopy sphere. We prove now that independently of $h$ the manifold $M^{n}(h)$ is diffeomorphic to the sphere. Indeed

$$
M^{n}(h)=S^{i} \times D^{j} \cup_{h^{\prime}} D^{i+1} \times S^{j-1} .
$$

\footnotetext{
${ }^{4}$ Part of this theorem was independently proven by Wallace who used similar ideas. The part of the theorem relative to the Poincaré conjecture was also proven by Stallings in a somewhat weaker form for $n \geq 7$ and by Zeeman for $n=5,6$. Zeeman used completely different methods.
} 
We now construct a diffeomorphism $M^{n}(h) \rightarrow S^{n}\left(S^{n}=S^{i} \times D^{j} \cup D^{i+1} \times S^{j-1}\right)$ : on the subset $D^{i+1} \times S^{j-1} \subset M^{n}(h)$ we take the identity diffeomorphism. Since the diffeomorphism $h^{\prime}: S^{i} \times D^{j} \rightarrow S^{i} \times D^{j}$ can be extended to a diffeomorphism $\bar{h}^{\prime}: S^{i} \times S^{j} \rightarrow S^{i} \times S^{j}$, the diffeomorphism given on $D^{i+1} \times S^{j-1} \subset M^{n}(h)$ can be extended to a map $M^{n}(h) \rightarrow S^{n}$, which has a "corner" at the common boundary

$$
S^{i} \times S^{j-1}=S^{i} \times D^{j} \cap D^{i+1} \times S^{j-1} .
$$

This corner is then smoothed, which proves the desired statement. Since $J_{i+j}=$ $B\left(S^{i+j}\right)$, any framing on $S^{i} \times S^{j}$ can be pulled back to a framing of $M^{n}(h)$ and the manifold $M^{n}(h)$ is diffeomorphic to $S^{n}$ for any $h$. This proves the lemma.

Let $\tilde{\theta}_{n} \subset G_{n}$ be the subgroups of elements representable by framed manifolds, which are combinatorially equivalent to the standard sphere (in $\S 3$ this group was denoted by $\operatorname{Im} \delta$, and some properties of these subgroups were given).

Lemma 9. Let $\alpha \in \tilde{\theta}_{n}$ and $\beta \in J_{i}, i \geq n$. Then $\alpha \beta \in J_{n+i}$, except when $i=n=$ $1,3,7$.

Proof. Consider the element $\alpha \beta \in G_{n+i}$. This element has a natural representation as a framed manifold $\tilde{S}^{n} \times S^{i}$, where $\alpha$ is represented by a framing of a Milnor sphere $\tilde{S}^{n}$. It turns out that for $i \geq n$ we have a diffeomorphism

$$
\tilde{S}^{n} \times S^{i}=S^{n} \times S^{i} .
$$

One can indeed prove that

$$
\tilde{S}^{n} \times D^{i+1}=S^{n} \times D^{i+1} .
$$

We consider the natural homeomorphism $\tilde{S}^{n} \rightarrow S^{n} \times D^{i+1}$ and approximate it by a smooth embedding $\tilde{S}^{n} \subset S^{n} \times D^{i+1} \subset R^{n+i+1}$ (see [3]). Because of the dimension restrictions, the smooth sphere $\tilde{S}^{n} \subset R^{n+i+1}$ has a trivial normal bundle. Therefore we get an embedding

$$
\tilde{S}^{n} \times D^{i+1} \subset S^{n} \times D^{i+1},
$$

where the sphere $\tilde{S}^{n} \times O$ is homotopic to the sphere $S^{n} \times O$. Using the results of Smale we conclude that

$$
\tilde{S}^{n} \times D^{i+1}=S^{n} \times D^{i+1},
$$

and therefore

$$
\tilde{S}^{n} \times S^{i}=S^{n} \times S^{i}
$$

Applying Lemma 8, and noting that $\alpha \beta \in B\left(S^{i} \times S^{n}\right)$ we arrive at the desired statement. The lemma is proved.

If the conditions of Lemma 9 are not satisfied, i.e., $i<n$, then for some $\alpha \in \tilde{\theta}_{n}$ and $\beta \in J_{i}$ we will possibly have $\alpha \beta \notin J_{i+n}$. Let $\alpha, \beta$ be a pair of just such elements, and let $\alpha$ be represented by the smooth framed sphere

$$
\tilde{S}^{n}=D_{1}^{n} \cup_{q} D_{2}^{2},
$$

where $q \in \operatorname{diff} S^{n-1}$. Moreover, let $\beta=J(h)$ where $h \in \pi_{i}(S O)$. Since

$$
\pi_{k}\left(S O_{k+2}\right)=\pi_{k}\left(S O_{\infty}\right)
$$

we can assume that $h \in \pi_{i}\left(S O_{n}\right)$. (We consider $\left.i \leq[n / 2]+1\right)$. Let us consider the smooth representative $f_{h}: S^{i} \rightarrow S O_{n}$ of the element $h$. The mapping $f_{h}$ induces the diffeomorphism

$$
f_{h}^{\prime}: S^{i} \times D^{n} \rightarrow S^{i} \times D^{n}
$$


which is defined by $f_{h}^{\prime}(x, y)=\left(x, f_{h}(x) \circ y\right)$. We can now consider the diffeomorphism $f_{h}^{\prime} \mid S^{i} \times S^{n-1}$ and the diffeomorphism $q f_{h}^{\prime} q^{-1}$ defined on $S^{i} \times S^{n-1}$ by the formula

where $S O_{n} \subset \operatorname{diff} S^{n-1}$.

$$
q f_{h}^{\prime} q^{-1}(x, y)=\left(x, q f_{h}(x) q^{-1} \circ y\right)
$$

We have the important

Lemma 10. If $\alpha \in \tilde{\theta}_{n}, \beta \in J_{i}$ and $\alpha \beta \notin J_{n+i}$, then the diffeomorphism

$$
q f_{n}^{\prime} q^{-1}: S^{i} \times S^{n-1} \rightarrow S^{i} \times S^{n-1}
$$

cannot be extended to a diffeomorphism

$$
S^{i} \times D^{n} \rightarrow S^{i} \times D^{n}
$$

( $h$, as before, is an element of $\left.J^{-1}(\beta), i<n\right)$.

Proof. We represent the element $\alpha \beta$ with the framed manifold $S^{i} \times \tilde{S}^{n}$ where $\tilde{S}^{n}=$ $D_{1}^{n} \cup_{q} D_{2}^{n}$. The set $B\left(S^{i} \times \tilde{S}^{n}\right)$ is easily seen to contain, beside $\alpha \beta$, the trivial element. Consider the sphere $S^{i} \times x_{0} \subset S^{i} \times \tilde{S}^{n}, x_{0} \in \tilde{S}^{n}$ and fix a field of $n$-frames $\tau_{0}^{n}$ normal to $S^{i} \times x_{0}$ in $S^{i} \times \tilde{S}^{n}$. We choose the field $\tau_{0}^{n}$ in such a way that the natural framing of $S^{i} \times \tilde{S}^{n}$ which determines the element $\alpha \circ 0=0$ should extend to the manifold

$$
B_{0}^{n+i+1}=\left[S^{i} \times \tilde{S}^{n} \times I(0,1 / 2)\right] \cup_{g_{0}} D^{i+1} \times D^{n} .
$$

Here the diffeomorphism

$$
g_{0}: \partial D^{i+1} \times D^{n} \rightarrow S^{i} \times \tilde{S}^{n} \times \frac{1}{2}
$$

is defined by the identity map onto the tubular neighborhood of the sphere $S^{i} \times x_{0} \subset$ $S^{i} \times \tilde{S}^{n}$ in the coordinates determined by the field $\tau_{0}^{n}$. To determine an analogous manifold

$$
B_{1}^{n+i+1}=\left[S^{i} \times \tilde{S}^{n} \times I\left(0, \frac{1}{2}\right)\right] \cup_{g_{1}} D^{i+1} \times D^{n},
$$

over which the framing of $S^{i} \times \tilde{S}^{n}$ defining $\alpha \beta$ could be extended, we use a field of frames $\tau_{1}^{n}$, which differs from the field $\tau_{0}^{n}$ by a smooth map $f: S^{i} \rightarrow S O_{n}$ which represents $h \in J^{-1}(-\beta) \subset \pi_{i}\left(S O_{n}\right)$. Now let

$$
\partial B_{0}^{n+i+1}=S^{i} \times \tilde{S}^{n} \cup M_{0}^{n+i}
$$

and

$$
\partial B_{1}^{n+i+1}=\tilde{S}^{n} \times S^{i} \times M_{1}^{n+i},
$$

where $M_{0}^{n+i}$ and $M_{1}^{n+i}$ are homotopy spheres. Since the trivial framing is pulled back to $M_{0}^{n+i}$ we get $M_{0}^{n+i} \in \theta^{n+i}(\partial \pi)$ and since $\alpha \beta \notin J_{n+i}, M_{1}^{n+i} \notin \theta^{n+i}(\partial \pi)$. Note that both manifolds $M_{0}^{n+i}$ and $M_{1}^{n+i}$ are composed of identical parts:

$$
\begin{aligned}
& M_{0}^{n+i}=\left[S^{i} \times \tilde{S}^{n} \backslash S^{i} \times \operatorname{Int} D_{1}^{n}\right] \cup_{g_{0}} D^{i+1} \times S^{n-1}, \\
& M_{1}^{n+i}=\left[S^{i} \times \tilde{S}^{n} \backslash S^{i} \times \operatorname{Int} D_{1}^{n}\right] \cup_{g_{1}} D^{i+1} \times S^{n-1},
\end{aligned}
$$

where

$$
g_{0}, g_{1}: S^{i} \times S^{n-1} \rightarrow S^{i} \times S^{n-1}, \quad g_{t}(x, y)=\left(x, g_{t x} \circ y\right), \quad g_{t x} \in S O_{n}, \quad t=0,1 .
$$

Moreover, the composition $g_{0}^{-1} g_{1}: S^{i} \times S^{n-1} \rightarrow S^{i} \times S^{n-1}$ is such that

$$
g_{0}^{-1} g_{1}(x, y)=(x, f(x) \circ y) \text {. }
$$


Let us begin to construct a homeomorphism $F: M_{1}^{n+i} \rightarrow M_{0}^{n+i}$. We establish a natural correspondence between the subsets $D^{i+1} \times S^{n-1} \subset M_{t}^{n+i}, t=0,1$, and try to extend it over

$$
Q=\left[S^{i} \times \tilde{S}^{n} \backslash S^{i} \times \operatorname{Int} D_{1}^{n}\right] \subset M_{t}^{n+i}, \quad t=0,1 .
$$

Since $\tilde{S}^{n}=D_{1}^{n} \cup_{q} D_{2}^{n}$, we have $Q=S^{i} \times D_{2}^{n}$. The diffeomorphism $F: M_{1}^{n+i} \rightarrow M_{0}^{n+i}$ is defined everywhere except on $Q \subset M_{1}^{n+i}$. Consider now the natural diffeomorphisms $d_{i}: S^{i} \times D_{2}^{n} \rightarrow Q \subset M_{t}^{n+i}$; on the boundary we have the composition

$$
S^{i} \times \partial D_{2}^{n} \stackrel{d_{1}}{\longrightarrow}\left[M_{1}^{n+i} \backslash \operatorname{Int} Q\right] \stackrel{F}{\longrightarrow}\left[M_{0}^{n+i} \backslash \operatorname{Int} Q\right] \stackrel{d_{0}^{-1}}{\longrightarrow} S^{i} \times \partial D_{2}^{n} .
$$

If the map $d_{0}^{-1} F d_{1}: S^{i} \times \partial D_{2}^{n} \rightarrow S^{i} \times \partial D_{2}^{n}$ could be extended to a map $S^{i} \times D_{2}^{n} \rightarrow$ $S^{i} \times D_{2}^{n}$, then the manifolds $M_{1}^{n+i}$ and $M_{0}^{n+i}$ would be diffeomorphic. However, they are not diffeomorphic. On the other hand, we have

$$
d_{0}^{-1} F d_{1}(x, y)=\left(x, q f q^{-1}(x) \circ y\right), \quad q \in \operatorname{diff} S^{n-1},
$$

and since $\tilde{S}^{n}=D_{1}^{n} \cup_{q} D_{2}^{n}, f(x) \in S O_{n}$. [If one considers the orthogonal transformation $p: S^{n-1} \rightarrow S^{n-1}$ in the natural coordinates of the sphere, induced by the coordinates of the ball $D_{1}^{n} \subset \tilde{S}^{n}$, then in the natural coordinates induced by $D_{2}^{n} \subset S^{n}$ it will have the form $q p q^{-1}$.] The lemma follows.

Lemma 10 easily implies

Theorem 1. Let $h \in \pi_{i}\left(S O_{n}\right)$ and $\beta=J(h)$. Moreover, let $\alpha \in \theta^{n}$ be represented by the framed manifold

$$
\tilde{S}^{n}=D_{1}^{n} \cup_{q} D_{2}^{n}, \quad q \in \operatorname{diff} S^{n-1} .
$$

If $\alpha \beta \notin J_{n+i}$ then the element $q h q^{-1} \in \pi_{i}\left(\operatorname{diff}^{0} S^{n-1}\right)$ does not belong to $\operatorname{Im} p_{1 *}\left(\pi_{i}\left(\operatorname{diff}^{0} D^{n}\right)\right), i<n$.

Proof. According to Lemma 10, the mapping $f: S^{i} \rightarrow S O_{n}$ has the property that the diffeomorphism $q f^{\prime} q^{-1} / S^{i} \times S^{n-1}$ does not extend to a diffeomorphism $S^{i} \times$ $D^{n} \rightarrow S^{i} \times D^{n}$. Therefore the element $q h q^{-1} \in \pi_{i}\left(\operatorname{diff}^{0} S^{n-1}\right)$ does not belong to $\operatorname{Im} p_{1 *}$ which is determined by smooth maps alone. However, the theorem now follows from the approximation lemma (see $\S 1$ ).

Consider now the homomorphisms

$$
\begin{array}{rlrl}
\lambda_{i}^{n}: & \pi_{i}\left(\operatorname{diff}^{0} S^{n-1}\right) & \rightarrow \pi_{0}\left(\operatorname{diff} S^{n+i-1}\right), \\
T: \pi_{0}\left(\operatorname{diff} S^{n+i-1}\right) & \rightarrow \Gamma^{i+n} \\
S: & \Gamma^{i+n} & \rightarrow \theta^{i+n} \\
\delta: & \theta^{i+n} & \rightarrow G_{n+i} / J_{n+i} .
\end{array}
$$

Let $q \in \pi_{0}\left(\operatorname{diff} S^{n-1}\right), h \in \pi_{i}\left(S O_{n}\right), i<n$.

Lemma 11. In the above notation

$$
[\delta S T(q)] \cdot J(h)= \pm \delta S T \lambda_{i}^{n}\left(q h q^{-1}\right) .
$$

Proof. Let us first show that the formula makes sense. Since $\delta S T(q) \in G_{n} / J_{n}$ and $i \leq(n / 2)+1$, the composition $[\delta S T(q)] \circ J(h)$ is, according to Lemma 9 , a unique element in the group $G_{n+i} / J_{n+i}$, i.e., where the right hand side of the formula is an element. What is the right hand side? Let us represent the element $h \in \pi_{i}\left(S O_{n}\right)$ 
by a map $f: D^{i} \rightarrow S O_{n}$, such that a neighborhood of the boundary of the ball is mapped into the identity of the group. Then the map

$$
q f q^{-1}: D^{i} \rightarrow \operatorname{diff}^{0} S^{n-1}
$$

will have the same property. Further consider the diffeomorphism

$$
S^{n-1} \times D^{i} \rightarrow S^{n-1} \times D^{i},
$$

generated by $q f q^{-1}$ and which is the identity on the boundary. As before we construct a diffeomorphism on $S^{i+n-1}$ : namely

$$
S^{i+n-1}=D^{n} \times S^{i-1} \cup S^{n-1} \times D^{i}
$$

and on $S^{n-1} \times D^{i} \subset S^{n+i-1}$ we have a diffeomorphism which is the identity on the boundary. This can then be naturally extended to $S^{n+i-1}$. The resulting diffeomorphism is nontrivial on $S^{n-1} \times D^{i} \subset S^{n+i-1}$ only, and belongs to the class $\lambda_{i}^{n}\left(q h q^{-1}\right)$. The homotopy sphere $S T \lambda_{i}^{n}\left(q h q^{-1}\right)$ is obtained by gluing together two balls $D_{1}^{n+i}$ and $D_{2}^{n+i}$ using this diffeomorphism. We write

$$
D_{l}^{n+i}=D_{l}^{n} \times D_{l}^{i}, \quad l=1,2 .
$$

Note that

$$
\partial\left(D_{l}^{n} \times D_{l}^{i}\right)=S^{n-1} \times D^{i} \cup D^{n} \times S^{i-1}
$$

and our diffeomorphism "rotates" each sphere $S_{x}^{n-i}$ for $x \in D_{l}^{i}$ by the diffeomorphism $q f q^{-1}(x)$. After we glue $D_{1}^{n+i}$ and $D_{2}^{n+i}$ together, each sphere $S^{n+1}=$ $S^{n-1} \times x$ will span two balls $D_{l}^{n} \times x, l=1,2$. The union $D_{1}^{n} \times D_{1}^{i} \cup D_{2}^{n} \times D_{2}^{i}$ along the part $D_{1}^{n} \times \partial D_{1}^{i}=D_{2}^{n} \times \partial D_{2}^{i}$, which is realized by the identity diffeomorphism (we should remember here that on that part of the sphere $S^{n+i-1}$ the diffeomorphism is the identity) is naturally diffeomorphic to $D^{n} \times S^{i}$. We now make the identification on the remaining part of the boundary, which is diffeomorphic to

$$
\partial D_{l}^{n} \times D_{l}^{i} / \partial D_{l}^{i} \times \partial D_{l}^{i}=S^{n-1} \times S^{i} .
$$

This is equivalent to gluing to $D^{n} \times S^{i}$, obtained after the first identification, the space $S^{n-1} \times D^{i+1}$ using the diffeomorphism

$$
F: S^{n-1} \times S^{i} \rightarrow S^{n-1} \times S^{i}
$$

for which

$$
F(x, y)=\left(q f q^{-1}(y) \circ x, y\right)
$$

(earlier we could have assumed that we were mapping not $D^{i}$ but $S^{i}$ into the groups $S O_{n}$ and $\operatorname{diff}^{0} S^{n-1}$, because the mappings took neighborhoods of the boundary into the identity of the group).

Hence the homotopy sphere $S T \lambda_{i}^{n}\left(q h q^{-1}\right)$ is obtained as follows: we take two manifolds $W_{1}=S^{n-1} \times D^{i+1}$ and $W_{2}=D^{n} \times S^{i}$ and identify their boundaries by the diffeomorphism

$$
F: S^{n-1} \times S^{i} \rightarrow S^{n-1} \times S^{i} .
$$

However, this manifold modulo $\theta^{n+i}(\partial \pi)$ can be constructed differently. We take the cartesian product $\tilde{S}^{n} \times S^{i}$, where $\tilde{S}^{n}=S T(q)$, and carry out a spherical modification on the cycle $x \times S^{i}, x \in \tilde{S}^{n}$, using the framing $\tau_{0}^{n}$, which is normal to $x \times S^{i}$ in $\tilde{S}^{n} \times S^{i}$ in such a way that the resulting manifold $M_{0}^{n+i}$ carries a framing cobordant to the trivial framing determining the zero element $0 \in B\left(\tilde{S}^{n} \times S^{i}\right)$; then we carry out another spherical modification, using $\tau_{1}^{n}$, in such a way that the resulting manifold carries a framing determining $[\delta S T(q)] \circ J(h)$. In the proof of Lemma 10 
we have shown that in this case the field $\tau_{1}^{n}$ differs from $\tau_{0}^{n}$ by a map $S^{i} \rightarrow S O_{n}$ representing the element $-h \in \pi_{i}\left(S O_{n}\right)$. As in the proof of Lemma 10, we attempt to construct a diffeomorphism $M_{1}^{n+i} \rightarrow M_{0}^{n+i}$ and come across the necessity of extending the map $F: S^{n-1} \times S^{i} \rightarrow S^{n-1} \times S^{i}$ over $D^{n} \times S^{i} \subset M_{1}^{n+i}$. However, on one hand the manifold $M_{0}^{n+i}$ is diffeomorphic to $S^{n+i} \bmod \theta^{n+i}(\partial \pi)$ and on the other hand the homotopy sphere $S T \lambda_{i}^{n}\left(q h q^{-1}\right)$ is constructed in such a way that if we attempt to construct a diffeomorphism of it onto $S^{n+i}$, then starting from the identity map on $D^{n} \times S^{i}$ we find that the extension of this over $S^{n-1} \times D^{i+1}$ necessitates that the same map

$$
F: S^{n-1} \times S^{i} \rightarrow S^{n-1} \times S^{i}
$$

be extended. The obstruction to extending the diffeomorphism $F$ over $S^{n-1} \times D^{i+1}$ occurs actually at a single point and is equal to some element $\gamma \in \Gamma^{n}$ for which

$$
\delta S(\gamma)=[\delta S T(q)] \cdot J(h)
$$

because the homotopy sphere representing the element $S(\gamma)$ coincides with the manifold $M_{1}^{n+i} \bmod \theta^{n+i}(\partial \pi)$. Therefore the manifolds $S T \lambda_{i}^{n}\left(q h q^{-1}\right)$ and $M_{1}^{n+i}$ are diffeomorphic mod $\theta^{n+i}(\partial \pi)$ and after applying $\delta$ the difference between them is lost. This completes the proof of the lemma.

We can now formulate our main results about the homotopy groups of the group of diffeomorphisms of a sphere.

Theorem 2. Let $h \in \pi_{i}\left(S O_{n}\right)$ with $i<n / 2+1$ and $\alpha \in \tilde{\theta}_{n} \subset G_{n}$ with $\alpha \circ J(h) \notin$ $J_{n+i}$. Consider any element $q \in \pi_{0}\left(\operatorname{diff} S^{n-1}\right)$ for which $\delta S T(q) \equiv \alpha\left(\bmod J_{n}\right)$. Then the elements

$$
\begin{gathered}
q h q^{-1} \in \pi_{i}\left(\operatorname{diff}^{0} S^{n-1}\right) \\
\mu_{i}^{n}\left(q h q^{-1}\right) \in \pi_{i-1}\left(\operatorname{diff}^{0} S^{n}\right), \ldots, \mu_{i-k}^{n+k} \circ \cdots \circ \mu_{i}^{n}\left(q h q^{-1}\right) \in \pi_{i-k+1}\left(\operatorname{diff}^{0} S^{n+k}\right)
\end{gathered}
$$

are nontrivial for all $0 \leq k \leq i-1$ and do not belong to $p_{1 *} \pi_{i-k-1}\left(\operatorname{diff}^{0} D^{n+k+1}\right)$.

Proof. Since the image of $\delta S T \lambda_{i}^{n}\left(q h q^{-1}\right)$ is nontrivial, the nontriviality of all those elements follows from Lemma 10 and the formula

$$
\lambda_{i}^{n}=\mu_{1}^{n+i-1} \circ \cdots \circ \mu_{i}^{n} .
$$

Let anyone of the described elements belong to $\operatorname{Im} p_{1 *}$. Since from the exact sequence (I) we have $\mu_{j}^{l}=\kappa_{*} \partial$ and $\operatorname{Im} p_{1_{8}}=\operatorname{Ker} \partial$, then any further application of $\mu_{j}^{l}$ (with corresponding values for $j$ and $l$ ) would annihilate that element. This gives a contradiction in all cases except when the last element, namely $\mu_{1}^{n+i+1} \circ \cdots \circ \mu_{i}^{n}\left(q h q^{-1}\right)$ belongs to $\operatorname{Im} p_{1 *}$. However, in this case the element would belong to $\operatorname{Ker} T$, which contradicts Lemma 10. The theorem is proved.

From Theorem 2 we immediately get

Corollary 1. If $\alpha \in \tilde{\theta}_{n} \subset G_{n}, \beta \in J_{i}, i<n / 2+1$ and $\alpha \beta \notin J_{i+n}$, then all groups $\pi_{i-k}\left(\operatorname{diff} S^{n+k-1}\right) / \operatorname{Im} p_{1 *}$ are nontrivial for $0 \leq k \leq i$.

Corollary 2. If, with the same assumptions, the element $\beta$ has prime order $p$, then all groups $\pi_{i-k}\left(\operatorname{diff} S^{n+k-1}\right) / \operatorname{Im} p_{1 *}$ contain at least $p$ elements (as before, $0 \leq k \leq i)$ 
Proof. The statement is obvious for $p=2$. If $p>2$, then it can be easily seen that the elements $q^{m} h q^{-m}$ and $q^{l} h q^{-l}$ are not equal $\bmod \operatorname{Im} p_{1 *}$ if $m-l \not \equiv 0(\bmod p)$. Note that

$$
\delta S T \lambda_{i}^{n}\left(q^{m} h q^{-m}\right)=m \delta S T \lambda_{i}^{n}\left(q h q^{-1}\right),
$$

i.e., the images of these elements are different for $m \not \equiv l(\bmod p)$. This gives the desired statement.

Some necessary data from tables:

1) $p=2$ :

$$
\begin{aligned}
& G_{1}=J_{1}=Z_{2}, \\
& G_{8}=Z_{2}+Z_{2}, \quad J_{8}=Z_{2}, \\
& G_{9}=Z_{2}+Z_{2}+Z_{2}, \quad J_{9}=Z_{2}, \\
& G_{1} G_{8}=J_{1} G_{8}=Z_{2}+Z_{2}, \\
& G_{8} J_{1} / J_{9}=Z_{2}, \\
& G_{10}=Z_{6}, \\
& J_{1} G_{9}=Z_{2}, \quad J_{10}=0, \\
& J_{1} G_{9} / J_{10}=Z_{2},
\end{aligned}
$$

2) $p>2$ :

$$
\begin{gathered}
G_{2 p-3}^{(p)}=J_{2 p-3}^{(p)}=Z_{p}, \\
G_{2(p-1) p-2}^{(p)} \neq 0, \quad J_{2 p(p-1)-2}^{(p)}=0, \\
\underbrace{G_{2 p(p-1)-2}^{(p)} \circ \cdots \circ G_{2 p(p-1)-2}^{(p)}}_{i \leq p-2 \text { copies }}{ }^{\circ J_{2 p-3}^{(p)} \not \subset \operatorname{Im} J .}
\end{gathered}
$$

\section{$\S 5$. RELATIONS BETWEen DIFFERENTIABLE AND LineAR SPHERE BUNDLES}

In this section we shall list the properties of smooth sphere bundles which are consequences of $\S 4$.

The universal sphere bundle has base $\operatorname{Pl}\left(S^{n-1}, R^{N}\right)$ for sufficiently large $N$, fiber $S^{n-1}$ and structure group diff $S^{n-1}$. From the fibration

$$
\operatorname{diff}\left(S^{n-1}, R^{N}\right) \stackrel{\text { diff } S^{n-1}}{\longrightarrow} P l\left(S^{n-1}, R^{N}\right)
$$

and the asphericity of the space $\operatorname{diff}\left(S^{n-1}, R^{N}\right)$ for dimensions $i$ with $2(i+n)+3<$ $N$, we conclude that

$$
\pi_{i-1}\left(\operatorname{diff} S^{n-1}\right) \approx \pi_{i}\left(P l\left(S^{n-1}, R^{N}\right)\right) .
$$

In particular, the group

$$
\pi_{i}\left(P l\left(S^{n-1}, R^{N}\right)\right)=\pi_{0}\left(\operatorname{diff} S^{n-1}\right)
$$

and hence is commutative. The action of the group $\pi_{0}\left(\operatorname{diff} S^{n-1}\right)$ on the groups $\pi_{i-1}\left(\operatorname{diff} S^{n-1}\right)$ via the transformation $q h q^{-1}$, where $q \in \pi_{0}\left(\operatorname{diff} S^{n-1}\right)$ and $h \in$ $\pi_{i-1}\left(\operatorname{diff} S^{n-1}\right)$ becomes under the isomorphism

$$
\pi_{i-1}\left(\operatorname{diff} S^{n-1}\right) \approx \pi_{i}\left(P l\left(S^{n-1}, R^{N}\right)\right) .
$$

The usual action of the fundamental group on the homotopy groups, and the orbits of $\pi_{1}\left(P l\left(S^{n-1}, R^{N}\right)\right)$ acting on $\pi_{i}\left(P l\left(S^{n-1}, R^{N}\right)\right)$ naturally correspond to the free homotopy classes of maps $S^{i} \rightarrow \operatorname{Pl}\left(S^{n-1}, R^{N}\right)$. It is known that a space is of simple homotopy type if the free homotopy classes of maps of spheres into the 
space coincide with the elements of homotopy groups. It follows from Theorem 1 that if $[\delta S T(q)] \circ J(h) \notin \operatorname{Im} J$, where $q \in \pi_{0}\left(\operatorname{diff} S^{n-1}\right)$ and $h \in \pi_{i-1}\left(S O_{n}\right) \subset$ $\pi_{i-1}\left(\operatorname{diff} S^{n-1}\right)$, then the element $q h q^{-1}$ does not belong to $\operatorname{Im} p_{1 *}$ and therefore does not belong to $\pi_{i-1}\left(S O_{n}\right) \subset \pi_{i-1}\left(\operatorname{diff} S^{n-1}\right)$. Thus $q h q^{-1} \neq h$.

Consequence 1. The space $\operatorname{Pl}\left(S^{n-1}, R^{N}\right)$ is not of simple homotopy type. The tables of the groups $G_{n}, \tilde{\theta}_{n}, J_{k}$ are given in [6] (except that instead of $G_{14}=Z_{2}+Z_{2}$ the erroneous $G_{14}=Z_{2}$ is given). In the same paper the multiplication table $G_{k} G_{l} \subset G_{k+l}$ is given. It follows from that table that one can take $i=1, n=8$; $i=1, n=9 ; i=2 p-3, n=2 k p(p-1)-2 k$ for all primes $p \geq 3$ and $k \leq p-2$. In the last case the element $J(h) \in J_{2 p-3}$ is of order $p$, which implies that for all $m=0,1, \ldots, p-1$ the elements $q^{m} h q^{-m}$ are distinct $\bmod \operatorname{Im} p_{1 *}$ (see Corollary 2, $\S 4)$.

For given values of pairs $(i, n)$ there exist the following kinds ${ }^{5}$ of smooth $S^{n-1}$ bundles over $S^{i+1}$ :

a) Bundles which are equivalent to $S O_{n}$-bundles in the group diff $S^{n-1}$ and are not equivalent to $S O_{n}$-bundles in the group $\operatorname{diff}^{0} S^{n-1}$. This follows from the fact that the free homotopy classes in $\mathrm{Pl}\left(S^{n-1}, R^{N}\right)$ do not coincide in dimensions $>1$ with the based homotopy classes (the fundamental group is commutative).

b) If we take, for example, $i=1, n=8$ then we can conclude that no element $q^{\prime} \in \pi_{0}\left(\operatorname{diff} S^{n-1}\right)$ will make

$$
q h q^{-1}-h \in \pi_{1}\left(\operatorname{diff}^{0} S^{n-1}\right)
$$

conjugate to an element of $\operatorname{Im} p_{1 *}$. This means that there exist smooth sphere bundles over spheres of dimension $>1$, which are not a boundary of a smooth ball bundle over the sphere.

Consequence 2. From Theorem 2 we conclude that for the same values of the pairs $(i, n)$ all the groups

$$
\pi_{i-k}\left(\operatorname{diff} S^{n+k-1}\right) / \operatorname{Im} p_{1 *}, \quad 0 \leq k \leq i,
$$

are nontrivial. This enables us to give a series of examples of nontrivial (nonlinearizable) bundles over $S^{i-k+1}$ with fiber $S^{n+k-1}$ and structure group $\operatorname{diff}^{0} S^{n+k-1}$ or diff $S^{n+k-1}$.

In conclusion the author wishes to express deep gratitude to D. V. Anosov, who pointed out to us the approximation lemma for the infinite-dimensional case (see Lemma 1). ${ }^{6}$

\section{Appendix 1. A problem of J. Muncrees}

Let $h \in \pi_{i}\left(S O_{k-1}\right) \subset \pi_{i}\left(S O_{k}\right)$ and $q \in \pi_{0}\left(\operatorname{diff} S^{k-1}\right)$. Then

$$
q h q^{-1}-h \in \pi_{i}\left(\operatorname{diff}^{0} S^{k-1}\right)
$$

and

$$
\lambda_{i}^{k}\left(q h q^{-1}-h\right) \in \pi_{0}\left(\operatorname{diff} S^{i+k-1}\right) .
$$

We define the transformation $\Phi: \pi_{i}\left(S O_{k-1}\right) \otimes \Gamma^{k} \rightarrow \Gamma^{k+i}$, by

$$
\Phi\left(h \otimes T_{q}\right)=T \lambda_{i}^{k}\left(q h q^{-1}-h\right),
$$

\footnotetext{
${ }^{5}$ If we pass to piecewise linear automorphisms of the sphere, all our bundles become equivalent to $S O$-bundles.

${ }^{6}$ Here we get nontrivial bundles even when $S O$-bundles do not exist.
} 
where $T: \pi_{0}\left(\operatorname{diff} S^{j-1}\right) \rightarrow \Gamma^{j}$. One can easily prove that the definition of $\Phi$ is correct. Lemma 11 and Theorem 2 give

Theorem 1.1. There exist numbers $i, k$ such that the homomorphism

$$
\Phi: \pi_{i}\left(S O_{k-1}\right) \otimes \Gamma^{k} \rightarrow \Gamma^{k+i}
$$

is nontrivial.

This theorem answers a problem of Muncrees (see [11]) and follows easily from the results of [6] which was published by the author before the problem was formulated.

\section{Appendix 2. Normal Bundles of HOMOtopy SPheres IN EUClidean SPACE}

The results of this appendix were obtained by the author in cooperation with L. N. Ivanovskiı̌. We consider a homotopy sphere $\tilde{S}^{n}$ embedded in $R^{n+j}$ with $j>(n / 2)+1$. It follows from [3] that such an embedding always exists and is unique up to a regular homotopy. Thus to any homotopy sphere $\tilde{S}^{n} \in \theta^{n}$ there corresponds a normal bundle in $R^{n+j}$ for $j>(n / 2)+1$; we denote this normal bundle by $H_{j}\left(\tilde{S}^{n}\right) \in \pi_{n-1}\left(S O_{j}\right) . H_{j}\left(S^{n}\right)$ is uniquely determined and depends additively on $\tilde{S}^{n} \in \theta^{n}$. We have thus the following homomorphisms:

$$
H_{j}: \theta^{n} \rightarrow \pi_{n-1}\left(S O_{j}\right), \quad j>\frac{n}{2}+1 .
$$
[4].

The following lemma is a simple consequence of the method used by Kervaire

Lemma 2.1. For $j>n / 2+1$ the kernel of the homomorphism $H_{j}$ contains the group $\theta^{n}(\partial \pi)$.

Proof. We can assume that $n$ is odd. The Milnor sphere $\tilde{S}^{n} \in \theta^{n}(\partial \pi)$ bounds a parallelizable $(n-1) / 2$-connected manifold $W^{n+1}$. This manifold can, according to Haefliger [3], be embedded in $R^{n+j}$ for the given values of $j$. The normal bundle of $W^{n+1}$ in $R^{n+j}$ is trivial; thus the normal bundle on the boundary $\tilde{S}^{n}=\partial W^{n+1}$ is also trivial. The lemma follows.

Remark. The spheres of $\theta^{n}(\partial \pi)$ can actually be embedded in $R^{n+2}$ with trivial normal bundle, but this does not affect our result.

Thus we have the homomorphism

$$
H_{j}: \theta^{n} / \theta^{n}(\partial \pi) \rightarrow \pi_{n-1}\left(S O_{j}\right), \quad j>\frac{n}{2}+1 .
$$

Let us consider the group $\pi_{n-1}\left(S O_{j}\right)$ and the Whitehead homomorphism $J: \pi_{n-1}\left(S O_{j}\right) \rightarrow \pi_{n+j-1}\left(S^{j}\right)$.

\section{Lemma 2.2 .}

$$
\operatorname{Ker} J \cap \operatorname{Tor} \pi_{n-1}\left(S O_{j}\right)=\operatorname{Im} H_{j},
$$

where $H_{j}: \theta^{n} / \theta^{n}(\partial \pi) \rightarrow \pi_{n-1}\left(S O_{j}\right)$ and $\operatorname{Tor} G$ is the finite part of the finitely generated abelian group $G$.

Proof. Since the Pontrjagin class of the homotopy sphere is trivial, we have

$$
\operatorname{Im} H_{j} \subset \operatorname{Tor} \pi_{n-1}\left(S O_{j}\right) \text {. }
$$


Since the normal bundle of a homotopy sphere in a euclidean space $R^{n+j}$ is determined by the element $\alpha \in \operatorname{Ker} J \subset \pi_{n-1}\left(S O_{j}\right)$, we have the inclusion

$$
\text { Ker } J \cap \operatorname{Tor} \pi_{n-1}\left(S O_{j}\right) \supset \operatorname{Im} H_{j} .
$$

We shall show that for $j>n / 2+1$ the inclusion going the opposite way holds. Let

$$
\alpha \in \operatorname{Ker} J \cap \operatorname{Tor} \pi_{n-1}\left(S O_{j}\right) .
$$

Consider the Thom complex $T_{j}(\alpha)$ of the $S O_{j}$-bundle over $S^{n}$ whose characteristic class is $\alpha$. The top homology cycle of $T_{j}(\alpha)$ has dimension $n+j$ and is spherical since $\alpha \in \operatorname{Ker} J$. We take the mapping of degree +1 of the sphere $S^{n+j} \rightarrow T_{j}(\alpha)$ and make it $t$-regular on $S^{n} \subset T_{j}(\alpha)$. Using spherical modifications, just as in [5] and [12], we find that the full preimage is of the same homotopy type as $S^{n}$, as long as $\alpha \in$ Tor $\pi_{n-1}\left(S O_{j}\right)$ and $j>n / 2+1$. This preimage is the homotopy sphere with normal bundle $\alpha$ and $S^{n+j}$ or $R^{n+j}$. This completes the proof.

Lemma 2.3. Let $\tilde{E}^{N}: \pi_{n+j-1}\left(S^{j}\right) \rightarrow G_{n-1} / J_{n-1}$ for $j>n / 2+1$ be the iterated suspension. Then Ker $\tilde{E}^{N}=\operatorname{Im} J$.

Proof. Obviously $J \pi_{n-1}\left(S O_{j}\right) \subset \operatorname{Ker} \tilde{E}^{N}$. Now let $\beta \in \operatorname{Ker} \tilde{E}^{N}$ be represented by a framed manifold $M^{n-1} \subset R^{n+j-1}$. Using the inequality $j>n / 2+1$ we find that $M^{n-1}$ is a homotopy sphere in the case when $n-1 \neq 4 k+2$ and $[(n-l) / 2-l]$ connected for $n-1=4 k+2$. In the second case, since $\beta \in \operatorname{Ker} \tilde{E}^{N}$, the Arf invariant will vanish and therefore we can again take $M^{n-1}$ to be a homotopy sphere; in both cases we obviously have $M^{n-1} \in \theta^{n-1}(\partial \pi)$. Therefore the manifold $M^{n-1}$ with any framing in $R^{n+j}$ determines an element of $J \pi_{n-1}\left(S O_{j}\right)$ because any embedding of the ordinary sphere in $R^{n+j-1}$ for $j>n / 2+1$ determines an element of $\operatorname{Im} J$. This proves the lemma.

Let us denote by $\tilde{\pi}_{n+j}\left(S^{j}\right) \subset \pi_{n+j}\left(S^{j}\right)$ the subgroup of elements which can be represented by framed homotopy spheres. If $j>n / 2+1$ then the subgroup $\tilde{\pi}_{n+j}\left(S^{j}\right)$ coincides with $\pi_{n+j}\left(S^{j}\right)$ for $n \neq 4 k+2$ and is of index $\leq 2$ for $n=4 k+2$. We also have

$$
\operatorname{Im} J \subset \tilde{\pi}_{n+j}\left(S^{j}\right)
$$

and

$$
\tilde{E}^{N} \tilde{\pi}_{n+j}\left(S^{i}\right) \subset \tilde{\pi}_{N+j+n}\left(S^{j+N}\right) / \operatorname{Im} J=\theta^{n} / \theta^{n}(\partial \pi) .
$$

From Lemmas 2.2 and 2.3 we get

Theorem 2.1. Consider the sequence of homomorphisms

$$
\rightarrow \theta^{n} / \theta^{n}(\partial \pi) \stackrel{H_{j}}{\longrightarrow} \pi_{n-1}\left(S O_{j}\right) \stackrel{J}{\rightarrow} \tilde{\pi}_{n+j-1}\left(S^{j}\right) \stackrel{\tilde{E}^{N}}{\longrightarrow} \theta^{n-1} / \theta^{n-1}(\partial \pi) \rightarrow \ldots,
$$

where $\tilde{\pi}_{n+j-1}\left(S^{j}\right)=\pi_{n+j-1}\left(S^{j}\right), n \neq 4 k+2$. This sequence has the property that $\operatorname{Im} H_{j}=\operatorname{Ker} J \cap \operatorname{Tor} \pi_{n-1}\left(S O_{j}\right)$ for $n \equiv 0 \bmod 4$, and it is exact in all other cases.

Proof. Lemmas 2.2 and 2.3 treat the exactness of this sequence at all terms except $\theta^{n-1} / \theta^{n-1}(\partial \pi)$. However, it is obvious that $\operatorname{Im} \tilde{E}^{N}=\operatorname{Ker} H_{j}$ since $\operatorname{Im} \tilde{E}^{N}$ consists exactly of those homotopy spheres $\tilde{S}^{n-1}$ which have trivial normal bundle in $R^{n+j-1}$. The theorem is proved.

Let us consider the homomorphism

$$
H_{j}: \theta^{n} / \theta^{n}(\partial \pi) \rightarrow \pi_{n-1}\left(S O_{j}\right), \quad j>\frac{n}{2}+1
$$

in greater detail. We have 
Theorem 2.2. The image of the homomorphism $H_{j}$ consists of elements of order $2^{S}$.

Proof. From Lemma 2.2 we know that $H_{j}\left(\theta^{n} / \theta^{n}(\partial \pi)\right)$ is contained in Ker $J \cap$ Tor $\pi_{n-1}\left(S O_{j}\right)$. Therefore the iterated inclusion homomorphism

$$
j_{N}: \pi_{n-1}\left(S O_{j}\right) \rightarrow \pi_{n-1}\left(S O_{j+N}\right)
$$

annihilates $\operatorname{Im} H_{j}$; this follows from the results of Adams [1] about the stable $J$ homomorphism. We first consider the case when $\alpha \in \operatorname{Im} H_{j}$ is such that $q \alpha=0$, where

$$
q: \pi_{n-1}\left(S O_{j}\right) \rightarrow \pi_{n-1}\left(S O_{j+1}\right) .
$$

In the fibration $S O_{j+1} / S O_{j}=S^{j}$ the element $\alpha$ belongs to the image of the homomorphism

$$
\partial: \pi_{n}\left(S^{j}\right) \rightarrow \pi_{n-1}\left(S O_{j}\right),
$$

since $j \alpha=0$ i.e., $\alpha=\partial \beta$. If $j$ is odd, then, for $n<2 j-2, \operatorname{Im} \partial$ consists of elements of order 2 alone and the proof is completed. If $j$ is even we use the well-known formula

$$
J \partial(\beta)=E^{l} \beta \circ\left[\tau_{j}, \tau_{j}\right],
$$

where $\beta \in \pi_{n}\left(S^{j}\right), n<2 j-2, \tau_{j}$ is the generator of the group $\pi_{j}\left(S^{j}\right)$ and [ , ] the Whitehead product. If $\beta$ is of odd order then

$$
E_{\beta}^{l} \circ\left[\tau_{j}, \tau_{j}\right] \neq 0,
$$

since $\left[\tau_{j}, \tau_{j}\right]$ is an element $\bmod C_{2}$ with Hopf invariant 2 , where $C_{2}$ is the class of finite 2-groups in the sense of Serre. This element can be handled like an element of Hopf invariant 1. This implies that the group Ker $J$, in the dimensions which we consider, consists of elements of order $2^{S}$. Since $\operatorname{Ker} q \subset \operatorname{Im} \partial$ we conclude that $\operatorname{Ker} q \cap \operatorname{Ker} J$ consists of elements whose order is of the form $2^{S}$.

We complete the proof by induction on $k$ : let $\operatorname{Ker} j_{k-1} \cap \operatorname{Ker} J$ be a group of order $2^{S}$. We now show the same for Ker $j_{k} \subset$ Ker $J$. Consider the homomorphism

$$
j_{k-1}: \operatorname{Ker} j_{k} \cap \operatorname{Ker} J \rightarrow \pi_{n-1}\left(S O_{j+k-1}\right) .
$$

The kernel of this homomorphism has, by induction, order $2^{S}$. The image lies in the intersection $\operatorname{Ker} q \cap \operatorname{Ker} J$; also $i+k-1>n / 2+1$ if $j>n / 2+1$ and $k>0$. Hence the image is also of order $2^{S}$ for some $S$.

Thus the homomorphism $H_{j}$ for $j>n / 2+1$ is trivial on the $p$-component of $\theta^{n}$ for $p>2$.

As an example consider the case when the homomorphism $H_{j}$ is nontrivial for $p=2$. Letting $n=16$ and using the tables in the work of Toda [13] one can deduce that $G_{16} / J_{16}=Z_{2}$ and the generator of this group cannot be decomposed in the form $G_{1} G_{15}$. Further consider $\pi_{28}\left(S^{12}\right), \pi_{29}\left(S^{13}\right)$; from the tables of Toda we find $G_{16} / J_{16} \neq E^{N} \pi_{29}\left(S^{13}\right)$. Using the previously obtained exact sequences we have the following theorem.

Theorem 2.3. ${ }^{7}$ The Milnor sphere $\tilde{S}^{16}$, which represents the nonzero element of the group $G_{16} / \mathrm{Im} J$, can be embedded in $R^{29}$ with nontrivial normal bundle.

\footnotetext{
${ }^{7}$ This theorem was proved in cooperation with L. N. Ivanovskil.
} 


\section{REFERENCES}

[1] J. F. Adams, On the stable J-homomorphism, Colloq. Algebraic Topology, 1962, Matematisk Institut, Aarhus Universitet, Aarhus, 1962. MR 26 \#3565.

[2] J. Cerf, Topologie de certains espaces de plongement, Bull. Soc. Math. France 89 (1961), 227-380. MR 25 \#3543.

[3] A. Haefliger, Differentiable embeddings, Bull. Amer. Math. Soc. 67 (1961), 109-112. MR 23 \#A665.

[4] M. Kervaire, Sur le fibré normal à une variété plongée dans l'espace euclidien. Bull. Soc. Math. France 87 (1959), 397-401. MR 22 \#5054.

[5] M. Kervaire and J. Milnor, Groups of homotopy spheres. I, Ann. of Math. (2) 77 (1963), 504-537. MR 26 \#5584.

[6] S. P. Novikov, Homotopy properties of the group of diffeomorphisms of the sphere, Dokl. Akad. Nauk SSSR 148 (1963), 32-35 = Soviet Math. Dokl. 4 (1963), 27-31. MR 26 \#1901.

[7] R. S. Palais, Local triviality of the restriction map for embeddings, Comment. Math. Helv. 34 (1960), 305-312. MR 23 \#A666.

[8] S. Smale, The generalized Poincaré conjecture in higher dimensions. Bull. Amer. Math. Soc. 66 (1960), 373-375. MR 23 \#A2220.

[9] , Diffeomorphisms of the 2-sphere, Proc. Amer. Math. Soc. 10 (1959), 621-626. MR $22 \# 3004$.

[10] T. E. Stewart, On groups of diffeomorphisms, Proc. Amer. Math. Soc. 11 (1960), 559-563. MR $22 \# 11400$.

[11] R. Lashof (Editor), Problems in differential and algebraic topology, Seattle Conference 1963, Ann. of Math. (2) 81 (1965), problem 4. MR 32 \#443.

[12] S. P. Novikov, A diffeomorphism of simply connected manifolds, Dokl. Akad. Nauk SSSR 143 (1962), 1046-1049 = Soviet Math. Dokl. 3 (1962), 540-543. MR 28 \#1628.

[13] H. Toda, Composition methods in homotopy groups of spheres, Ann. of Math. Studies, no. 49, Princeton Univ. Press, Princeton, N. J., 1962. MR 26 \#777. 